\title{
Femtocell power control methods based on users' context information in two-tier heterogeneous networks
}

\author{
Reben Kurda ${ }^{*}$, Lila Boukhatem ${ }^{1 *}$ and Megumi Kaneko2*
}

\begin{abstract}
In this paper, we consider a two-tier macrocell/ femtocell overlaid heterogeneous network based on orthogonal frequency division multiple access (OFDMA) technology. Although the co-channel spectrum allocation provides larger bandwidth for both macrocell and femtocells, the resulting cross-tier interference may prevent macrocell users in the vicinity of femtocells to achieve their minimum required signal-to-interference plus noise ratio (SINR) in downlink. Therefore, we propose femtocell power control strategies for mitigating the interference experienced by macrocell users while preventing the femtocell throughput degradation. In particular, the proposed power control schemes make use of femto and macro users' context information in terms of positioning for setting the appropriate prioritization weights among the current victim macro users and the femto users in outage. System-level simulations show that our schemes enhance the throughput of macrocell users while maintaining a high performance for femtocell users compared to a conventional power allocation. Moreover, we show that the proposed prioritization weights allow to achieve the required level of macrocell/femtocell throughput trade-off.
\end{abstract}

Keywords: Heterogeneous networks; Macrocell; Femtocell; Power control; Interference management

\section{Introduction}

One of the main goals of the $4 \mathrm{G}$ long-term evolution advanced (LTE-A) system is to enhance the capacity of mobile radio networks and to improve the quality of service (QoS) levels experienced by prospective users [1]. Towards this end, LTE-A introduces the deployment of heterogeneous networks (HetNets), whereby low power nodes such as femtocell base stations (FBS)s are overlaid within a large macrocell served by a macrocell base station (MBS). Generally, FBSs provide several advantages such as offering high performance to their users due to the proximity between transmitters and receivers, and all the benefits of a backhaul connection (such as fiber optic or digital subscriber line DSL) which can be used to connect to the operator's macrocell. Moreover, FBSs, which are mainly deployed indoor, provide capacity offload to

\footnotetext{
*Correspondence: Reben.Kurda@|ri.fr; Lila.Boukhatem@lri.fr; meg@i.kyoto-u.ac.jp

${ }^{1}$ Laboratoire de Recherche en Informatique (LRI), University of Paris-Sud 11 - CNRS, 15, rue Georges Clémenceau, 91405 Orsay Cedex, France

${ }^{2}$ Graduate School of Informatics, Kyoto University, Yoshida-Honmachi, Sakyo-ku, Kyoto 606-8501, Japan
}

outdoor users or macrocell user equipments (MUEs), and some studies foresee that in future communication scenarios, almost $50 \%$ of voice and $70 \%$ of data traffic are likely to be generated indoors [2]. However, the crosstier interference from the transmitting FBSs becomes a crucial issue for MUEs in the vicinity of femtocell coverage areas, as they may observe a severe degradation of their signal-to-interference plus noise ratio (SINR) level [3].

Several research works have addressed the crosstier interference problem in two-tier HetNets based on orthogonal frequency division multiple access (OFDMA) transmission. This major issue can be addressed through adapted resource block (RB) allocation schemes at the scheduling level and/or evolved time, frequency, or power control techniques [1]. All these approaches propose to mitigate the cross-tier-interference on victim MUEs and can be classified as centralized, assistance-based, or decentralized solutions.

The centralized approaches rely on a central entity and a global knowledge of the parameters related to the different link gains whose transmission may induce extensive

\section{Springer}

(c) 2015 Kurda et al: licensee Springer. This is an Open Access article distributed under the terms of the Creative Commons Attribution License (http://creativecommons.org/licenses/by/4.0), which permits unrestricted use, distribution, and reproduction in any medium, provided the original work is properly credited. 
signaling and generate long delays. In the assistance-based solutions, the MBS supports the FBSs in making their resource usage decisions using some global information and/or the reported measurements from MUEs/FUEs. In this category, the proposed power control solutions focus on adjusting dynamically the transmission power of FBSs given the evolving interference situation. In [4], the interfered victim MUEs report their reference signal received power (RSRP) with the corresponding cell's physical identity and channel quality indicator (CQI) to the macrocell who identifies the interfering (aggressor) femtocells and informs them about the interference situation. By adjusting the FBSs' transmit power, macrocells could ensure a low interference to their MUEs at the expense of a throughput degradation of femtocell users.

In [5], we proposed an MBS-assisted power adjustment strategy for interference mitigation in a mobility environment where macrocells and femtocells are co-located. The key benefits of this scheme were to achieve higher performance for nonclosed-subscriber group (CSG) macrocell users, as well as avoiding potential service degradation of femtocell users by taking into account mobile user locations and SINR at both macro- and femtocell sides. An extended approach was proposed in [6] to define two power adjustment strategies with different degrees of awareness of femtocell users' performance.

Another set of approaches consider the concept of clustering of femtocells for the power adjustment. In [7], the femtocell's initial power setting is correlated to the number of active femtocells in the cluster. Both MBS-assisted and distributed sensing are proposed to derive the number of active small cells.

Other assistance-based approaches address the interference issue from a frequency dimension basis. In [8], the frequency resources of the macrocell are reused by the femtocells while relying on an adapted spectrum scheduling. The key idea of the proposed scheme is that femtocells avoid using the same frequency resources of the nearby MUEs according to the sensing process performed independently by them and the scheduling scheme carried out by the MBS.

An intra-cell handover (IHO) scheme applied to both macrocell and femtocells is proposed in [9] to mitigate the cross-tier interference. The scheme relies on a received signal strength -based power control mechanism when the IHO scheme fails in getting free sub-channels in the interfering femtocell. [10] proposed a hybrid resource coordination for a mixed macrocell and femtocell deployment, where the MBS can notify the femtocell to release the interfered allocated resources relieving the victim MUE from interference. Even if the proposals in [9] and [10] improve the performance of MUEs, they cause nonnegligible throughput deteriorations to FUEs. It is shown that a proper combination of $\mathrm{IHO}$ and power control techniques reduces the outage probability for nonsubscribers compared to that of closed and open access.

Another category of approaches consider decentralized solutions for interference mitigation in OFDMA femtocell deployment. Most of the solutions involve a joint optimization of the power allocation and the channel allocation or spectrum assignment. The authors in [11] proposed a self-organized framework of femtocells which autonomously perform the spectrum assignment and transmission power based on users' reported measurements. The study evaluated the gain in spectral efficiency and power consumption for the cases with and without macrocell interference.

In [12-15], decentralized power allocation approaches are proposed under the assumption of limited MUE channel information. In the neighbor-friendly scheme of [12], FBSs allocate the total adjusted power on sub-channels in order to maintain targeted FUEs' sum rates while mitigating the interference on MUEs. However, some assumptions about channel stability over several frames may not be valid especially in mobility scenarios. In [13], the effect of different macrocell sizes on the power control is analyzed when assuming femtocells' overhearing ability of MUEs' RSRP measurements. Downlink (DL) power allocation methods are proposed in $[14,15]$, where no control information exchange is assumed between the MBS and FBSs. Instead, each FBS predicts the RB allocation to its close-by MUEs based on their overheard channel state information (CSI) feedback from MUEs to MBS. Based on this MUE allocation prediction, each FBS can perform resource allocation to FUEs while mitigating interference towards close-by MUEs. However, interference mitigation among FBSs is not considered.

Finally, [16] showed that the use of radio environment maps (REM) information increases the efficiency of practical power control mechanisms of femtocells. The authors showed that under unknown shadowing terms, the use of statistical information retrieved from the REM database leads to a performance close to the ideal case.

In this paper, we mainly focus on power control approaches that take benefit from the radio resource control (RRC) signaling messages, and especially the RSRP signal $[5,6,15]$, to mitigate the downlink interference using an MBS-assisted power adjustment strategy. Our key objective is to improve the spectral efficiency of MUEs, while preserving a high throughput to FUEs. More specifically, we propose two context-based adjustment schemes whose common objective is to mitigate the interference on victim MUEs while implementing two different degrees of awareness of FUEs' throughput degradation. Our two adaptive power control schemes, called respectively global power adjustment (GPA) and selective power adjustment 
(SPA), differ in their selection strategy of the set of FBSs executing the power adjustment as well as the amount of power to reduce. The main advantage of our proposed approach over traditional ones is that it is based on power adjustment parameters whose values are dynamically adapted to the interference impact of each femtocell on the global interference situation. To estimate such femtocell's liability on the global interference, we use a Score Function which quantifies this value based not only on the RSS feedback returned by MUEs but also on some context information (such as the number and location of MUEs/FUEs) retrieved from a REM database. Exploiting this context information about users' number and localization enriches the decision policy and brings added knowledge value to the power allocation decision process. Finally, we discuss and analyze the effects of the Score Function weighting parameters on the global system performance in terms of macrocell and femtocell throughput.

The remainder of this paper is organized as follows. Section 2 describes the system model and main assumptions. Section 3 presents the proposed power adjustment schemes based on both users' context factors and dynamic power adjustment parameters. In Section 4, the simulation environment and results are presented and discussed. Finally, Section 5 concludes the paper.

\section{System model and assumptions}

We consider a macrocell/femtocell overlay system in which $L$ femtocells are geographically positioned in streets located at the edge of the coverage area of one MBS referred as $M$ (see Figure 1). We assume that $I$ MUEs are uniformly distributed within the MBS coverage area and are not allowed to access a neighboring femtocell $F_{l},(l=$ $1, \ldots, L)$ when they approach its coverage area. In other words, all the outdoor users are considered non-CSG users for $F_{l}$ 's operator. Each $F_{l}$ serves $J$ FUEs uniformly distributed in its coverage area. The transmissions are OFDMA-based and all MUEs and FUEs share the same frequency band divided in $K$ RBs. We also assume that MBS $M$ is fully loaded and cannot execute any intrachannel allocation to mitigate the potential interference caused to MUEs. To perform the resource allocation of their RBs, both MBS and FBSs require their user equipments (UEs) to periodically report their signal quality expressed by the CQI in terms of SINR. The received DL SINR of a MUE $i$ associated with MBS $M$ on RB $k(k=$ $1, \ldots, K)$ can be expressed as

$$
\operatorname{SINR}_{M, \mathrm{MUE}_{i}}^{k}=\frac{\frac{p_{M}^{k}}{\mathrm{PL}_{M, \mathrm{MUE}_{i}}}\left|G_{M, \mathrm{MUE}_{i}}^{k}\right|^{2}}{\sum_{F_{l} \in \mathcal{Z}_{i, k}} \frac{p_{F_{l}}^{k}}{P L_{F_{l}, \mathrm{MUE}_{i}}}\left|G_{F_{l}, \mathrm{MUE}_{i}}^{k}\right|^{2}+N_{0}}
$$

where $p_{M}^{k}$ is the current transmit power allocated on RB $k$ by the serving cell MBS $M$ and $\left|G_{M, M_{i}}^{k}\right|$ is the channel fast fading gain between MBS $M$ and $\mathrm{MUE}_{i}$ on RB $k$. Similarly, $p_{F_{l}}^{k}$ is the transmit power of neighboring femtocell $F_{l}$ on $\mathrm{RB} k$, while $\mathcal{Z}_{i, k}$ represents the set of all interfering FBSs on user $\mathrm{MUE}_{i}$ on RB $k$. Similarly, $\left|G_{F_{l}, \mathrm{MUE}_{i}}^{k}\right|$ is the channel fast fading gain between $\mathrm{MUE}_{i}$ and FBS $F_{l}$ on $\mathrm{RB} k . \mathrm{PL}_{M, \mathrm{MUE}_{i}}$ and $\mathrm{PL}_{F_{l}, \mathrm{MUE}_{i}}$ represent the pathloss from $\mathrm{MUE}_{i}$ 's serving cell MBS $M$ and FBS $F_{l}$ respectively, and $N_{0}$ is the power of the additive white Gaussian noise.

Similarly, the DL $\mathrm{SINR}_{F_{l}, \mathrm{FUE}_{j}}^{k}$ of a given $\mathrm{FUE}_{j}$ associated with a femtocell $F_{l}$ on RB $k$ can be expressed as:

$\mathrm{SINR}_{F_{l}, \mathrm{FUE}_{j}}^{k}=\frac{\frac{p_{F_{l}}^{k}}{\mathrm{PL}_{F_{l}, \mathrm{FUE}_{j}}}\left|G_{F_{l}, \mathrm{FUE}_{j}}^{k}\right|^{2}}{N_{0}+A_{j}^{k}+\sum_{\substack{F_{m} \in \mathcal{V}_{j, k} \\ m \neq l}} \frac{p_{F_{m}}^{k}}{\mathrm{PL}_{F_{m}, \mathrm{FUE}_{j}}}\left|G_{F_{m}, \mathrm{FUE}_{j}}^{k}\right|^{2}}$,

where $p_{F_{l}}^{k}$ is the current transmit power allocated on RB $k$ by the serving femtocell $F_{l}$ and $\left|G_{F_{l}, \mathrm{FUE}_{j}}^{k}\right|$ is the channel fast fading gain between $\mathrm{FUE}_{j}$ and its serving femtocell $F_{l}$. $p_{F_{m}}^{k}$ is the transmit power of a neighboring $\mathrm{FBS} F_{m}$, while $\mathcal{V}_{j, k}$ represents the set of all interfering FBSs on user $\mathrm{FUE}_{j}$ on RB $k .\left|G_{F_{m}, \mathrm{FUE}_{j}}^{k}\right|$ is the channel fast fading gain between $\mathrm{FUE}_{j}$ and its neighboring FBS $F_{m} . \mathrm{PL}_{F_{l}, \mathrm{FUE}_{j}}$ stands for the pathloss between $\mathrm{FUE}_{j}$ and $\mathrm{FBS} F_{l}$ while $A_{j}^{k}$ is the interference generated by MBS $M$ on resource block $k$, namely,

$$
A_{j}^{k}=\frac{p_{M}^{k}}{\mathrm{PL}_{M, \mathrm{FUE}_{j}}}\left|G_{M, \mathrm{FUE}_{j}}^{k}\right|^{2}
$$

For our system model needs, we define the following parameters:

- $\mathrm{SINR}_{\mathrm{MUE}}^{\text {target }}$ : the predefined SINR requirement threshold of each $\mathrm{MUE}_{i}$ and each allocated RB $k$.

- SINR $_{\mathrm{FUE}}^{\text {target }}$ : the predefined SINR requirement threshold for each $\mathrm{FUE}_{j}$ and each allocated RB $k$.

- $\mathrm{RSS}_{M, \mathrm{MUE}_{i}}^{k}$ and $\mathrm{RSS}_{F_{l}, \mathrm{MUE}_{i}}^{k}$ : the received signal strength by $\mathrm{MUE}_{i}$ from MBS $M$ and FBS $F_{l}$ on RB $k$, respectively [17]. They are expressed as:

$$
\begin{aligned}
\mathrm{RSS}_{M, \mathrm{MUE}_{i}}^{k} & =\frac{p_{M}^{k}}{\mathrm{PL}_{M, \mathrm{MUE}_{i}}}, \\
\mathrm{RSS}_{F_{l}, \mathrm{MUE}_{i}}^{k} & =\frac{p_{F_{l}}^{k}}{\mathrm{PL}_{F_{l}, \mathrm{MUE}_{i}}} .
\end{aligned}
$$




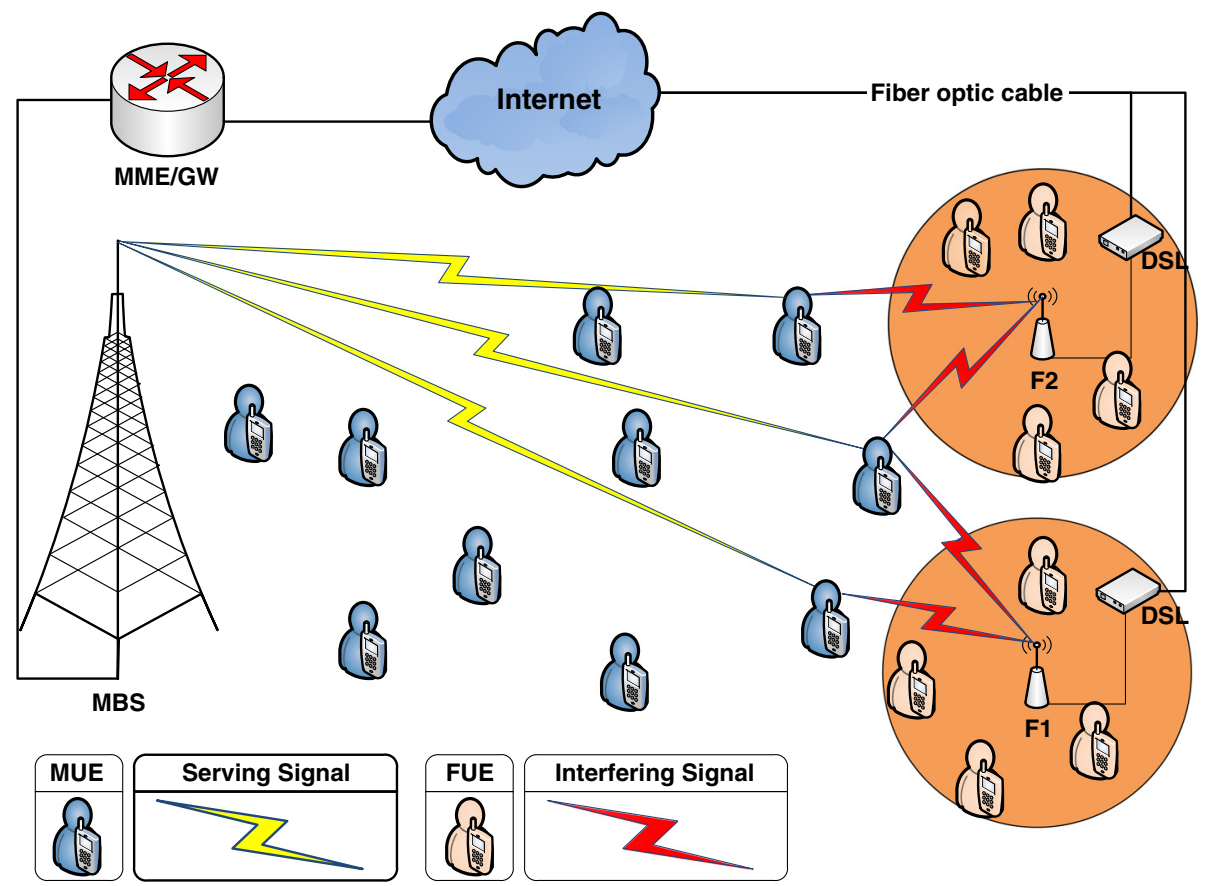

Figure 1 Two-tier heterogeneous network.

- $\triangle$ SINR: a positive protection margin, expressed in $\mathrm{dBs}$, used together with SINR $\mathrm{MUE}_{\mathrm{MU}}^{\text {target }}$ to determine if $\mathrm{MUE}_{i}$ experiences a bad serving channel. This is confirmed if the following condition is verified:

$$
\operatorname{SINR}_{M, \mathrm{MUE}_{i}}^{k}<\mathrm{SINR}_{\mathrm{MUE}}^{\mathrm{target}}+\Delta \mathrm{SINR} .
$$

- $\Delta P$ : a positive power protection margin, measured in $\mathrm{dBs}$ and used with the reported $\mathrm{RSS}_{F_{l}, \mathrm{MUE}_{i}}^{k}$ to check whether $\mathrm{RSS}_{M, \mathrm{MUE}_{i}}^{k}$ from the serving MBS $M$ is indicating a pending connection loss. This is verified if the following condition is true:

$$
\mathrm{RSS}_{M, \mathrm{MUE}_{i}}^{k}<\mathrm{RSS}_{F_{l}, \mathrm{MUE}_{i}}^{k}+\Delta P .
$$

Note that $\Delta P$ and $\triangle$ SINR values should be adequately tuned to avoid a potential oscillatory behavior. For example, if $\Delta P$ is too low, $\mathrm{MUE}_{i}$ may suffer too long from the interfering $F_{l}$ before MBS $M$ initiates the interference mitigation strategy. Inversely, if $\Delta P$ is too high, MBS $M$ activates the interference mitigation procedure even if the interference from neighboring $F_{l}$ is insubstantial, which leads to an increase in the signaling overhead.

Moreover, in this paper, we take benefit from the REM concept as a support to our interference mitiga- tion techniques. The term REM is generally used to refer to a database (local and/or global) which gathers location-dependent information about the environment where the radio system operates [18]. The REM concept is useful to develop optimized resource allocation and enhanced inter-cell interference coordination (eICIC) solutions that require to capture the evolution of random processes such as the channel quality, user mobility, and traffic dynamics. Therefore, we propose in this paper to make use of local REM databases at both MBS and femtocell sides to store local information. More specifically, the MBS REM registers all the RSSs recently fed back by the MUEs to inform about the interference condition caused by the neighboring FBSs on their respective allocated RBs when conditions (6) and (7) are fulfilled. Moreover, the context parameters (to be defined in the next section) will also be recorded in each local REM to help the power adjustment decisions. The REM databases are also used to store other less dynamic parameters such as $\operatorname{SINR}_{\mathrm{MUE}}^{\text {target }}, \mathrm{SINR}_{\mathrm{FUE}}{ }^{\text {target }}, \triangle \mathrm{SINR}$ and $\Delta P$ values.

\section{Femtocell power adjustment problem}

\subsection{Formulation of the optimization problem}

Since the femtocells are deployed in the macrocell coverage area with full frequency reuse, the transmission signal 
from FBSs will generate a strong interference to neighboring MUEs, causing a large degradation of their received $\mathrm{SINR}_{M, \mathrm{MUE}_{i}}^{k}$. The goal of the considered femtocell power adjustment is to maximize the sum of the macrocell and femtocells' throughputs, while satisfying simultaneously MUEs' and FUEs' SINR requirements.

Since it is assumed that all RBs are fully loaded, i.e., they are all allocated to MUEs beforehand, the considered throughput optimization problem can be formulated as:

$$
\begin{aligned}
& \max _{p_{F_{l}}^{k}} \sum_{k=1}^{K} \sum_{F_{l}} \tau_{F_{l}}^{k}\left(p_{F_{l}}^{k}\right)+\tau_{M}^{k}\left(p_{F_{l}}^{k}\right) \\
& \text { s.t. } \operatorname{SINR}_{M, \mathrm{MUE}_{i}}^{k} \geq \operatorname{SINR}_{\mathrm{MUE}}^{\text {target }}+\Delta \mathrm{SINR} \\
& \forall i \in\{1, \ldots, I\}, \forall k \in \mathcal{K}_{i}, \\
& \operatorname{SINR}_{F_{l}, \mathrm{FUE}_{j}}^{k} \geq \operatorname{SINR}_{\mathrm{FUE}}^{\text {target }} \\
& \forall j \in\{1, \ldots, J\}, \forall l \in\{1, \ldots, L\}, \forall k \in \mathcal{K}_{j}, \\
& p_{F_{l}}^{k} \geq 0 \quad \forall F_{l} \in\{1, \ldots, L\}, \forall k \in\{1, \ldots, K\} \\
& \sum_{k=1}^{K} p_{F_{l}}^{k} \leq P_{F l}^{\mathrm{Max}} \quad \forall k \in\{1, \ldots, K\}
\end{aligned}
$$

where $\mathcal{K}_{i}$ denotes the set of RBs allocated to $\mathrm{MUE}_{i}, \mathcal{K}_{j}$ the set of RBs allocated to $\mathrm{FUE}_{j}$, and $P_{F_{l}}^{\mathrm{Max}}$ denotes the maximum transmit power of femtocell $F_{l} \cdot \tau_{F_{l}}^{k}$ and $\tau_{M}^{k}$ are the throughput of the femtocell and macrocell user, respectively, in $\mathrm{RB} k$, and which can be expressed as:

$$
\begin{aligned}
\tau_{M}^{k}\left(p_{F_{l}}^{k}\right) & =\mathrm{B}_{M}^{k} \cdot\left(1-\operatorname{BLER}\left(\operatorname{SiNR}_{M, \mathrm{MUE}_{i}}^{k}\left(p_{F_{l}}^{k}\right)\right)\right), \\
\tau_{F_{l}}^{k}\left(p_{F_{l}}^{k}\right) & =\mathrm{B}_{F_{l}}^{k} \cdot\left(1-\operatorname{BLER}\left(\operatorname{SINR}_{F_{l}, \mathrm{FUE}_{j}}^{k}\left(p_{F_{l}}^{k}\right)\right)\right), \\
\mathrm{B}_{u}^{k} & =\Omega \cdot b_{u}^{k} \cdot \frac{N_{\mathrm{RB}} S_{\mathrm{RB}}}{t_{\mathrm{RB}}}, u \in\left\{M, F_{l}\right\}
\end{aligned}
$$

where $\Omega$ is a fixed parameter that depends on the network configuration, $b_{u}^{k}$ is the number of bits/symbol depending on the modulation and coding scheme selected by $\mathrm{MUE}_{i}$ for $u=M$ and by $\mathrm{FUE}_{j}$ for $u=F_{l} . N_{\mathrm{RB}}$ and $S_{\mathrm{RB}}$ are the number of data subcarriers and symbols per $\mathrm{RB}$, respectively. Finally, $t_{\mathrm{RB}}$ is the $\mathrm{RB}$ time duration [19].

Note that similar optimization problems were studied in $[12,20]$ and $[21]$. While $[20,21]$ considered only power minimization problem, maximization of the weighted sum of femtocell and macrocell throughput was investi- gated in [12]. However, only one femtocell was considered and no QoS constraints such as minimum SINR were introduced. Even for that simplified problem, it was shown that the optimization problem is nonconvex with respect to the femtocell power allocation parameters $p_{F}^{k}$, making it difficult to find the global optimum. Thus, the global optimization of our problem by a centralized approach may be even more difficult and intractable. Therefore, we propose a heuristic approach for semidistributed femtocell power control to achieve a good balance between the macro- and femtocells' performances. Note that, as the optimal solution cannot be derived under our dynamic environment assumptions, we will derive the upper bounds of the macro- and femtocells' throughputs to get a better idea about the performance of our sub-optimal solution.

\subsection{Proposed power control strategies}

In our model, various MUEs are randomly positioned within MBS M's coverage and each active $\mathrm{MUE}_{i}$ periodically sends to MBS $M$ a CQI in terms of $\operatorname{SINR}_{M, M^{\prime}}^{k} E_{i}$ as expressed in Equation 1 and experienced on each allocated RB $k$. Before performing the resource allocation procedure based on the adopted scheduling strategy, MBS $M$ first checks the quality of MUEs' communication channels based on the returned CQI [3-5].

For all MUEs and for all their allocated RBs for which condition (6) is fulfilled, MBS $M$ asks the concerned users to send a measurement report (MR) in terms of RSSs of the received pilot signals from their current serving cell and all neighboring femtocells. The RSS reports help MBS $M$ to identify the source of interference and to check whether it is caused by neighboring cells or if it is a consequence of any other effect such as fast fading or noise. For this purpose, condition (7) is checked to determine if for a given $\mathrm{MUE}_{i}$, the signal received from the neighboring femtocell $F_{l}$ covers the signal of its serving macrocell. If so, MBS $M$ identifies $F_{l}$ as an interfering cell for victim $\mathrm{MUE}_{i}$ and $F_{l}$ is added to the set of interfering FBSs on $\mathrm{MUE}_{i}, \mathcal{Z}_{i, k}: \mathcal{Z}_{i, k} \leftarrow \mathcal{Z}_{i, k} \cup\left\{F_{l}\right\}$. To keep track of the $\mathrm{RSS}_{F_{l}, \mathrm{MUE}_{i}}^{k}$ evolution perceived from each interfering FBS, MBS makes use of a local REM table to record the latest reported $\mathrm{RSS}_{F_{l}, \mathrm{MUE}_{i}}^{k}$ values.

In order to mitigate the interference generated by each interfering FBS in its local REM table, MBS $M$ initiates the power adjustment decision process as follows. First, for each victim $\mathrm{MUE}_{i}$, MBS identifies all the RBs $k$ on which $\mathrm{MUE}_{i}$ suffers from interference (i.e., those which fulfill condition (7)). Then, it retrieves from the REM table the set of femtocells causing this interference on $\mathrm{MUE}_{i}$ on RB $k$ (i.e., the set $\mathcal{Z}_{i, k}$ ).

The main objective of our approach is to express, for each interfering femtocell, its impact level on the interference situation. To do so, we define the Score Function 
$f\left(F_{l}\right)$ for each interfering femtocell $F_{l}$ in the REM table as follows:

$$
f\left(F_{l}\right)=\theta_{1} R_{F_{l}}+\theta_{2} N_{F_{l}}-\theta_{3} Q_{F_{l}}
$$

where $R_{F_{l}}, N_{F_{l}}$ and $Q_{F_{l}}$ are the context parameters defined as follows:

- $\mathcal{R}_{F_{l}}$ : the set of MUEs impacted by femtocell $F_{l}$ only (i.e., which verify condition (7)) and $R_{F_{l}}$ denotes its cardinality,

$$
\begin{aligned}
\mathcal{R}_{F_{l}} & =\left\{\mathrm{MUE}_{i}, i=1, \ldots, I ; \exists k \in \mathcal{K}_{i}, \mathcal{Z}_{i, k}=\left\{F_{l}\right\}\right\}, \\
R_{F_{l}} & =\left|\mathcal{R}_{F_{l}}\right|
\end{aligned}
$$

- $\mathcal{N}_{F_{l}}$ : the set of MUEs located in the overlapping areas between $F_{l}$ and any neighboring $F_{m}$, and $N_{F_{l}}$ denotes its cardinality,

$$
\begin{aligned}
& \mathcal{N}_{F_{l}}=\left\{\mathrm{MUE}_{i}, i=1, \ldots, I ; \exists F_{m} \in \mathcal{L}, \exists k \in \mathcal{K}_{i},\right. \\
& \left.\mathcal{Z}_{i, k} \supseteq\left\{F_{l}\right\} \underset{m \neq l}{\cup}\left\{F_{m}\right\},\left|\mathcal{Z}_{i, k}\right| \geqslant 2\right\}, \\
& N_{F_{l}}=\left|\mathcal{N}_{F_{l}}\right| .
\end{aligned}
$$

- $\mathcal{Q}_{F_{l}}$ : the set of FUEs observing an outage after decreasing the power of their serving cell $F_{l}$ and $Q_{F_{l}}$ denotes its cardinality,

$$
\begin{aligned}
& \mathcal{Q}_{F_{l}}=\left\{\mathrm{FUE}_{j}, j=1, \ldots, J ; \operatorname{SINR}_{F_{l}, \mathrm{FUE}_{j}}^{k}<\operatorname{SINR}_{\mathrm{FUE}}^{\text {target }}\right\}, \\
& Q_{F_{l}}=\left|\mathcal{Q}_{F_{l}}\right| .
\end{aligned}
$$

Finally, $\theta_{i},(i=1, \ldots, 3)$ are the positive weighting factors summing up to 1 and reflecting the priorities of the context parameters.

The context parameters refer to the number of victim MUEs and FUEs as well as their respective locations within the interference area. By assigning different weights to the MUEs affected by only one FBS and those affected by multiple FBSs in Equation 12, we differentiate the impact of those two types of interference situations in the Score Functions. Thus, these context parameters will help in identifying the set of femtocells which have the greater and shared impact on a given interference situation and hence allow for an adapted and coordinated power adjustment proportional to this impact. Figure 2 illustrates a simple scenario in which both interference situations are observed.

The Score Function $f\left(F_{l}\right)$ defined in Equation 12 can be either positive or negative. A positive $f\left(F_{l}\right)$ score means that the associated femtocell $F_{l}$ impacts more importantly the MUE than the FUEs under its coverage and thus has the ability to tolerate more reduction of its transmission power. Conversely, a negative score value reflects a serious outage situation of $F_{l}$ 's FUE. Note that $f\left(F_{l}\right)$ is evaluated at each frame as long as the interference lasts. Its value will vary according to the power adjustment strategy to be applied in the current frame as well as the mobility of MUEs.

The main objective now is to estimate the exact amount of power to be used by each interfering femtocell for the adjustment purpose. To resolve this problem, we propose two power adjustment strategies baptized global power adjustment (GPA) and selective power adjustment (SPA). GPA and SPA offer a different degree of awareness of FUE performance and consequently impacts the MUE performance as well due to the tradeoff between their conflicting requirements in terms of spectral efficiency. More specifically, GPA and SPA implement a different selection mechanism of the interfering femtocells which are to apply the power adjustment and the amount of the power adjustment (increase or decrease). Besides, the associated power adjustment parameters to be introduced hereafter offer an additional leverage in managing the compromise between FUE and MUE performances.

For each victim $\mathrm{MUE}_{i}$ on $\mathrm{RB} k$, MBS $M$ retrieves from the REM table the set of femtocells responsible for this interference and determines their respective Score Function values. Then, MBS $M$ finds the maximum interference $\mathrm{MI}_{\mathrm{MUE}_{i}}^{k}$ which can be allowed by a victim $\mathrm{MUE}_{i}$ on RB $k$ as:

$$
\mathrm{MI}_{\mathrm{MUE}_{i}}^{k}=\frac{\mathrm{RSS}_{M, \mathrm{MUE}_{i}}^{k}}{\operatorname{SINR}_{\mathrm{MUE}}^{\text {target }}}-N_{0} .
$$

Afterwards, MBS $M$ computes for each interfering $F_{l}$ in the REM table the new value of its transmission power $p_{F_{l}}^{\prime}$ in $\mathrm{RB} k$ as:

$$
p_{F_{l}}^{\prime k}=\alpha_{F_{l}}^{k} \cdot \beta_{F_{l}}^{k} \cdot p_{F_{l}}^{k}
$$

where we have

$$
\beta_{F_{l}}^{k}=\frac{\mathrm{MI}_{\mathrm{MUE}_{i}}^{k}}{\sum_{l=1}^{L} \mathrm{RSS}_{F_{l}, \mathrm{MUE}_{i}}^{k}} .
$$

$p_{F_{l}}^{k}$ represents the latest transmitted power of the interfering $F_{l}$ in $\mathrm{RB} k$, and $\mathrm{RSS}_{F_{l}, \mathrm{MUE}_{i}}^{k}$ is the measured received signal strength from $F_{l}$ by $\mathrm{MUE}_{i}$ for the allocated RB $k$. First, the previous power level $p_{F_{l}}^{k}$ is adjusted by the parameter $\beta_{F_{l}}^{k}$ which represents the allowed perfemtocell interference level, where $\mathrm{MI}_{\mathrm{MUE}_{i}}^{k}$ has been normalized by the sum of all RSSs of interfering femtocells. Hence, $\beta_{F_{l}}^{k}$ is the same for all $F_{l}$ and the received RSS by $\mathrm{MUE}_{i}$ after this adjustment becomes

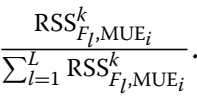




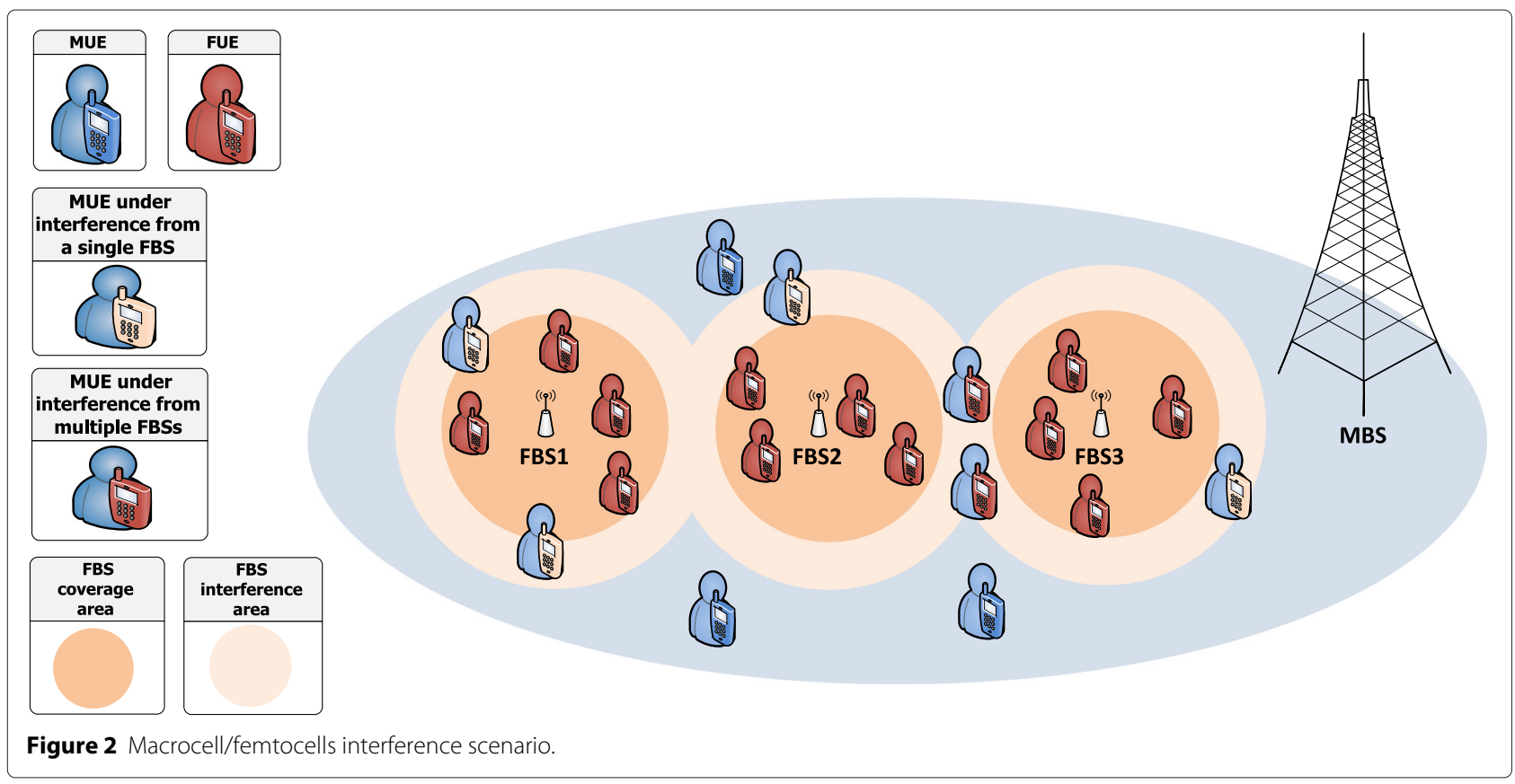

Next, the resulting power is further adjusted by the boosting factor $\alpha_{F_{l}}^{k}$ whose value is proportional to $f\left(F_{l}\right)$ score. Using the boosting factor $\alpha_{F_{l}}^{k}$ enables to adapt the power allocation proportionally to the femtocells' role in the interference situation rather than uniformly as usually performed in traditional power adjustment approaches. However, the amount of power boosting will be limited by a tunable system parameter $x,(0<$ $x \leq 1$ ) which represents the maximum allowed power reduction/increase ratio of the nominal power $\beta_{F_{l}}^{k} p_{F_{l}}^{k}$, i.e., $p_{F_{l}}^{\prime} \in\left[\beta_{F_{l}}^{k} p_{F_{l}}^{k}(1-x), \beta_{F_{l}}^{k} p_{F_{l}}^{k}(1+x)\right]$. Therefore, we have:

$$
\begin{cases}1-x<\alpha_{F_{l}}^{k} \leq 1 & \text { if } f\left(F_{l}\right) \geqslant 0 \\ 1<\alpha_{F_{l}}^{k} \leq \min \left(1+x, \frac{P_{F_{l}}^{\mathrm{Max}}}{\beta_{F_{l}}^{k} p_{F_{l}}^{k}}\right) & \text { otherwise, }\end{cases}
$$

Moreover, the $\alpha_{F_{l}}^{k}$ value for each $F_{l}$ should be inversely proportional to its corresponding score $f\left(F_{l}\right)$. Indeed, the higher the $f\left(F_{l}\right)$ score, the greater the impact of $F_{l}$ on the MUE interference so the more it should reduce its interference by setting a lower $\alpha_{F_{l}}^{k}$, with $1-x \leq \alpha_{F_{l}}^{k} \leq 1$. On the contrary, a lower $f\left(F_{l}\right)$ value implies a larger $\alpha_{F_{l}}^{k}$. In particular, the case $f\left(F_{l}\right)<0$ means that the proportion of unsatisfied FUEs is greater than that of victim MUEs, and hence $\alpha_{F_{l}}^{k}$ should be increased within the range $\left[1, \min \left(1+x, \frac{P_{F_{l}}^{\mathrm{Max}}}{\beta_{F_{l}}^{k} p_{F_{l}}^{k}}\right)\right]$. Thus, we propose the following setting of $\alpha_{F_{l}}^{k}$ in each case:

$$
\begin{cases}1-\min \left(x, \frac{f\left(F_{l}\right)}{\max _{i, k} f\left(F_{l}\right)}\right) & \text { if } f(F l) \geqslant 0 \\ \min \left(1+\min \left(x, \frac{f\left(F_{l}\right)}{\mathcal{Z}_{i, k} f\left(F_{l}\right)}\right), \frac{P_{F_{l}}^{\mathrm{Max}}}{\beta_{F_{l}}^{k} p_{F_{l}}^{k}}\right) & \text { otherwise, }\end{cases}
$$

where $\frac{f\left(F_{l}\right)}{\max _{\mathcal{Z}_{i, k}}\left(F_{l}\right)}$ and $\frac{f\left(F_{l}\right)}{\min _{i, k}\left(F_{l}\right)}$ act as the weights for FBS $F_{l}$ when $f\left(F_{l}\right)$ is positive or negative, respectively.

At this step, the selection of the set of the interfering femtocells that are to apply the boosting factor $\alpha_{F_{l}}^{k}$ defines the power adjustment strategy. As mentioned above, we propose two adjustment strategies: GPA and SPA.

\subsubsection{Global power adjustment}

In the GPA strategy, we propose to apply the power adjustment using the boosting factors to all the interfering femtocells. It is worth noticing here, that GPA strategy acts on all femtocells participating in the interference whatever their Score Function values. Therefore, GPA will create a balance between the offered throughput to victim MUEs and the performance of FUEs which may suffer from the power adjustment (see Algorithm 1). 


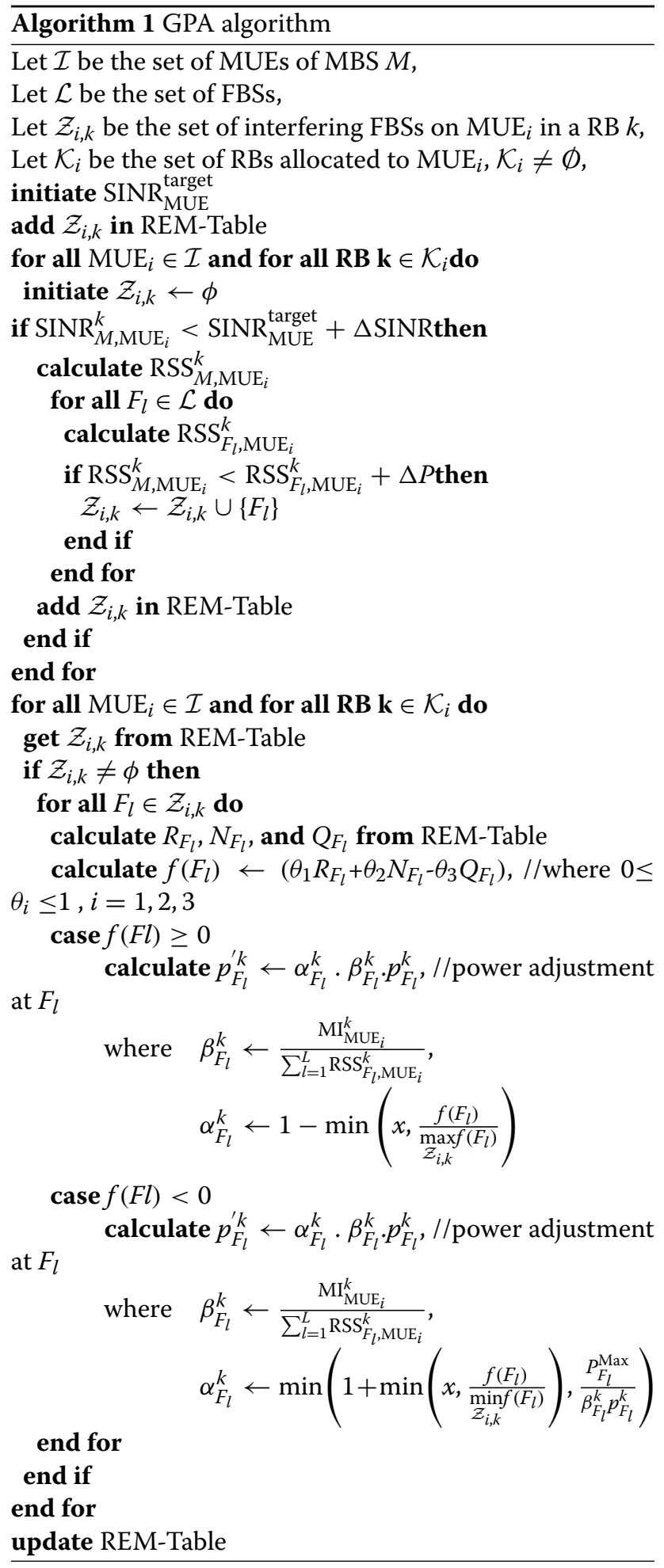

\subsubsection{Selective power adjustment}

The aim of this second strategy is to apply $\alpha_{F_{l}}^{k}$ adjustment parameters only for those femtocells which show a positive Score Function $\left(f\left(F_{l}\right) \geq 0\right)$. The rest of interfering femtocells $\left(f\left(F_{l}\right)<0\right)$ are allocated a fixed and neutral parameter value $\alpha_{F_{l}}^{k}=1$. Such a strategy will give more priority to MUEs in terms of throughput since femtocells suffering from a severe FUE outage situation will not benefit from an adapted power reduction proportional to their 'suffering' level. As a consequence, in SPA strategy, $\alpha_{F_{l}}^{k}$ are derived as follows:

$$
\left\{\begin{array}{cl}
1-\min \left(x, \frac{f\left(F_{l}\right)}{\max _{i, k}\left(F_{l}\right)}\right) & \text { if } f\left(F_{l}\right) \geqslant 0 \\
\alpha_{F_{l}}^{k}=1 & \text { otherwise }
\end{array}\right.
$$

Similarly to GPA, the adjustment process in SPA is repeated recursively at each frame until the interference caused to $\mathrm{MUE}_{i}$ on RB $k$ fades.

\subsection{Required signaling information exchange between cells}

The required signaling information between the macrocell and femtocells may be exchanged through two main possibilities: i) the backhaul connection using the core network infrastructure (optic fiber), through the S1 interface and femtocell gateway $[22,23]$ and ii) the direct X2 interface between macrocells and femtocells [3]. These two communication means will impact differently the performance mainly in terms of delay and signaling overhead.

The X2 interface enables the support of resource management functionalities operating in short timescales such as interference, mobility, and handover [23]. Therefore, the X2 interface is more suitable to support the signaling exchange in our power adjustment strategy as the S1 interface will enduce longer delays. In fact, power adjustments and several context information (i.e., RSS, SINR, ..., etc ) have to be periodically exchanged between the MBS and femtocell local REMs, and the delay imposed by the interface should be at a shorter timescale compared to this periodicity in order to allow a real-time response of the operations.

As regards to the signaling load, the amount of exchanged information becomes more stringent as the number of femtocells increases. In this respect, the backhaul connection may provide enough bandwidth for the signaling overhead but introduces harsh delay constraints, making it difficult to satisfy the real-time requirements [24]. It is worth noticing that the coordination between cells in our proposed scheme requires a limited signaling load for each updating cycle, namely:

- the measurement report $\mathcal{Q}_{F_{l}}$ which represents the number of $F_{l}$ 's FUEs in outage situation. This message is triggered by $F_{l}$ and sent to the corresponding MBS.

- the values of $p_{F_{l}}^{\prime k}$ for each RB $k$ and each $F_{l}$, to be sent by the MBS to the corresponding FBS $F_{l}$. 


\section{Performance evaluation}

\subsection{Simulation environment}

To investigate the performance of our proposed power allocation mechanisms, we implemented various simulation scenarios using LTE-Sim simulator [19]. The network topology consists of one MBS $M$ and several neighboring FBSs positioned in a street with a reasonable distance from $M$. Each FBS is placed in a building and serves a maximum of four users registered as CSG users and moving at a low speed compared to the outdoor users (3 vs. $30 \mathrm{~km} / \mathrm{h}$ ). MUEs are randomly positioned within the macrocell and move according to a random walk mobility model constrained by the street layout of $30 \mathrm{~m}$. The main simulation parameters are detailed in Table 1.

We used path loss models as specified in 3GPP TSG WG4 [17] with an external wall attenuation of $20 \mathrm{dBm}$. In a first set of experiments, the Score Function's weighting parameters $\theta_{1}, \theta_{2}$, and $\theta_{3}$ have a fixed value of $0.3,0.3$, and 0.4 , respectively. Note that the effect of these weighting parameters will be analyzed in Section 4.3.

\subsection{Performance of the power adjustment strategies}

In this first set of experiments, we evaluate the performance of our power allocation schemes using extensive simulations and gain further insight on the influence of the involved parameters on the macrocell/femtocell throughput performance. Regarding the scheduling module, we used the reference modified largest weighted delay first (MLWDF) algorithm. All MUEs/FUEs in the cell support three different types of application flows: CBR, VoIP,

Table 1 Simulation parameters

\begin{tabular}{|c|c|}
\hline Parameters & Values \\
\hline Number of macrocells & 1 \\
\hline Number of femtocells & 30 \\
\hline Number of MUEs & 10 to 60 \\
\hline Number of FUEs & 4 \\
\hline Bandwidth & $10 \mathrm{MHz}$ \\
\hline Total number of RBs & 50 \\
\hline Macro Tx power & $43 \mathrm{dBm}$ \\
\hline Femto Tx power & $23 \mathrm{dBm}$ \\
\hline Thermal noise density & $-174 \mathrm{dBm} / \mathrm{Hz}$ \\
\hline Carrier frequency & $3.5 \mathrm{GHz}$ \\
\hline UE noise figure & $2.5 \mathrm{~dB}$ \\
\hline Macrocell radius & $1 \mathrm{~km}$ \\
\hline FUEs' average speed & $3 \mathrm{~km} / \mathrm{h}$ \\
\hline MUEs' average speed & $30 \mathrm{~km} / \mathrm{h}$ \\
\hline $\operatorname{SINR}_{\mathrm{MUE}}^{\text {target }}$ & $5.7 \mathrm{~dB}$ \\
\hline SINR & $12.6 \mathrm{~dB}$ \\
\hline$\Delta P$ & $0.1 \mathrm{~dB}$ \\
\hline
\end{tabular}

and Video. CBR traffic is generated at a rate of 5-byte packets/s, whereas the VoIP flows generate 20-byte packets/s using an ON/OFF model [25]. The Video service is a 242 kbps data source with H.264 coding [26].

Quality of service in terms of throughput is measured for our proposed power allocation strategies GPA and SPA and compared with the Basic scheme where no power adjustment strategy is considered, as well as the Reference scheme proposed in [9], and which can be viewed as a classical femtocell power adjustment scheme that is unaware of the FUEs' performance. Note that in the Basic scheme, the femtocells transmit at their maximum transmit power on each RB. In other words, the Basic scheme corresponds to the upper bound of the FUEs' achievable throughput. The Basic and Reference schemes are noted 'Basic' and 'Ref.' in the figures, respectively. In order to better estimate the quality of our obtained suboptimal solution, we have also evaluated the MUEs' throughput upper bound denoted UP-Bound, which indicates the maximum achievable throughput for MUEs in the absence of femtocells, i.e., without any interference. Finally, we have compared our algorithms to the eICIC method based on almost blank subframes (ABS), denoted ABS in the legends. In this solution, the femtocells mute their transmission (of control and data signals) to enable the scheduling of victim MUEs by the macrocell, hence avoiding the cross-tier interference. We applied one of the ABS patterns considered in 3GPP with a duty cycle of $1 / 8$ where femtocells remain silent during $1 / 8$ of each subframe [27].

Figure 3 shows the average per-user throughput of MUEs for the supported CBR flows. We can easily observe that for all the schemes, the throughput degrades with the number of active MUEs as this number is correlated to the generated traffic. Moreover, we can also see that the gain in throughput for our proposed strategies is higher than for the Basic and Ref. strategies especially when the number of users is increased from 30 to 60. Our GPA and SPA strategies offer a gain of almost $12 \%$ and $16 \%$, compared to the Basic scheme, and $9 \%$ and $14 \%$ compared to the Reference scheme, respectively. The same behavior is observed for VoIP flows (Figure 4) which shows almost a similar performance gain. This increase in throughput for higher user density is mainly due to an increased probability of interference situations when MUEs approach the femtocells' coverage. Figure 3 also shows that SPA strategy performs better than GPA since it gives a higher priority to MUEs, providing them a higher average throughput. We can also observe that SPA strategy achieves the closest performance to the MUEs' throughput upper bound. Both proposed schemes outperform all reference schemes in terms of MUEs' throughput.

Figures 5 and 6 illustrate FUEs' average per-user throughput for CBR and VoIP flows, when varying the 


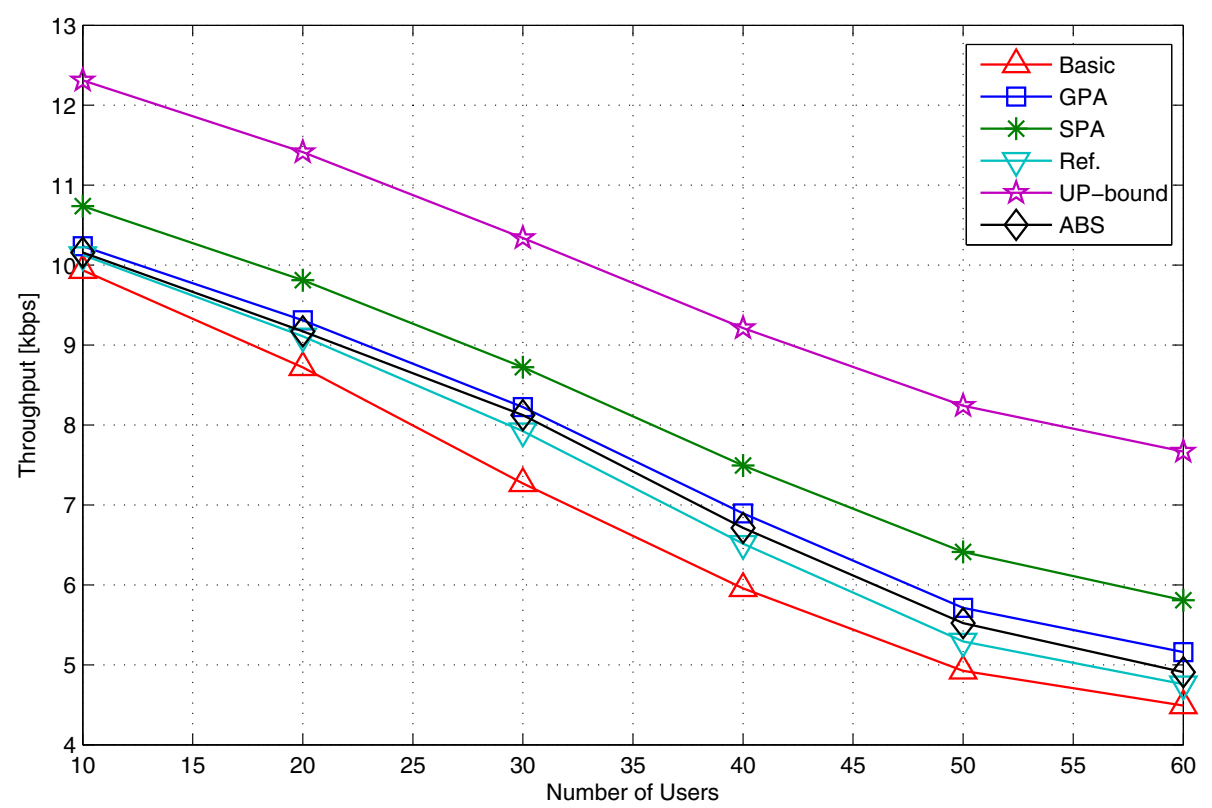

Figure 3 Average per-user throughput of MUEs for CBR flows.

number of active MUEs. As the power adjustment in general degrades the transmission power of femtocells to mitigate the interference effect on victim MUEs, the GPA scheme offers as expected a better average throughput for FUEs. However, we observe a similar FUE throughput performance for both SPA and Reference schemes, while ABS has the worst performance. Figures 5 and 6 show that the throughput degradation compared to the Basic scheme is only $5 \%$ and $7 \%$ as regards to GPA and SPA, respectively. Note that in the Basic scheme, the femtocells transmit at their maximum transmit power on each RB.

Figure 7 shows the average per-user throughput of MUEs for the supported Video flows. We have an improvement of $13 \%$ to $18 \%$ for SPA and $11 \%$ to $15 \%$ for

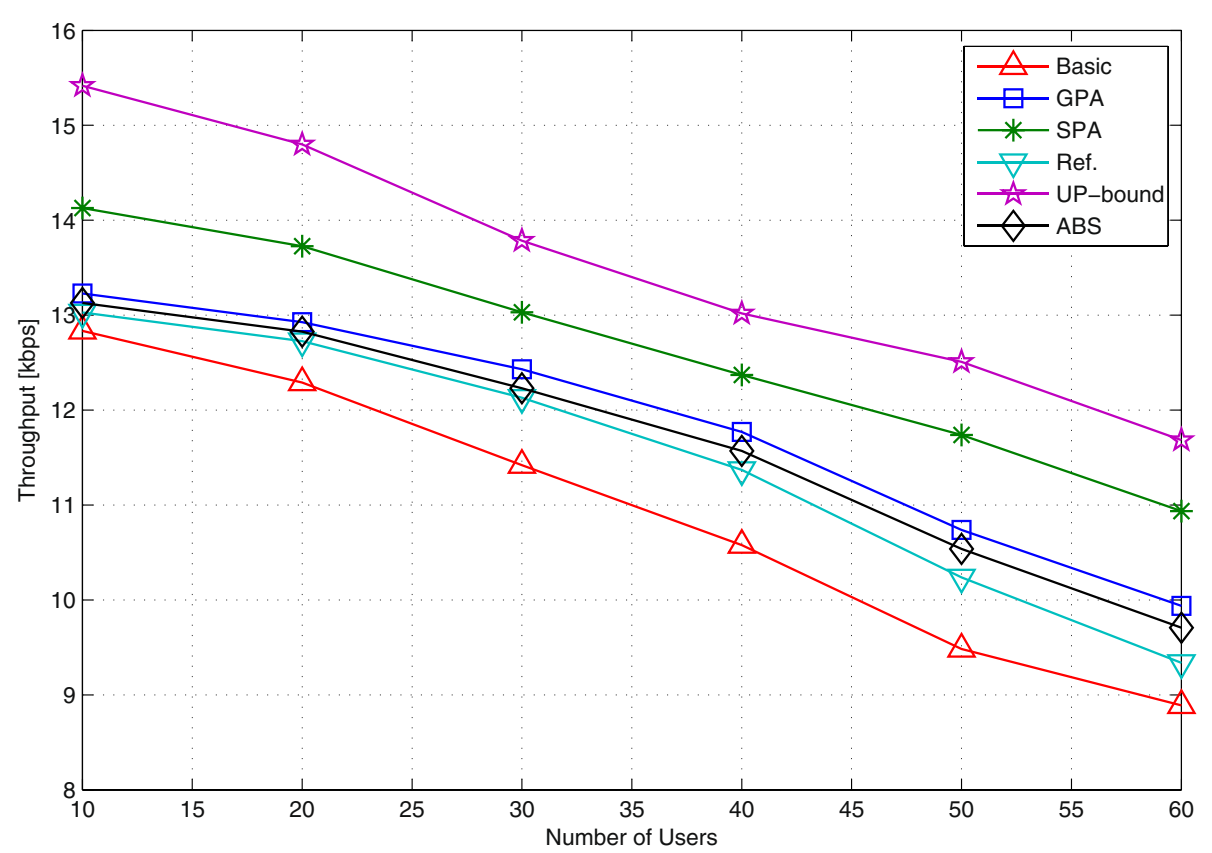

Figure 4 Average per-user throughput of MUEs for VolP flows. 


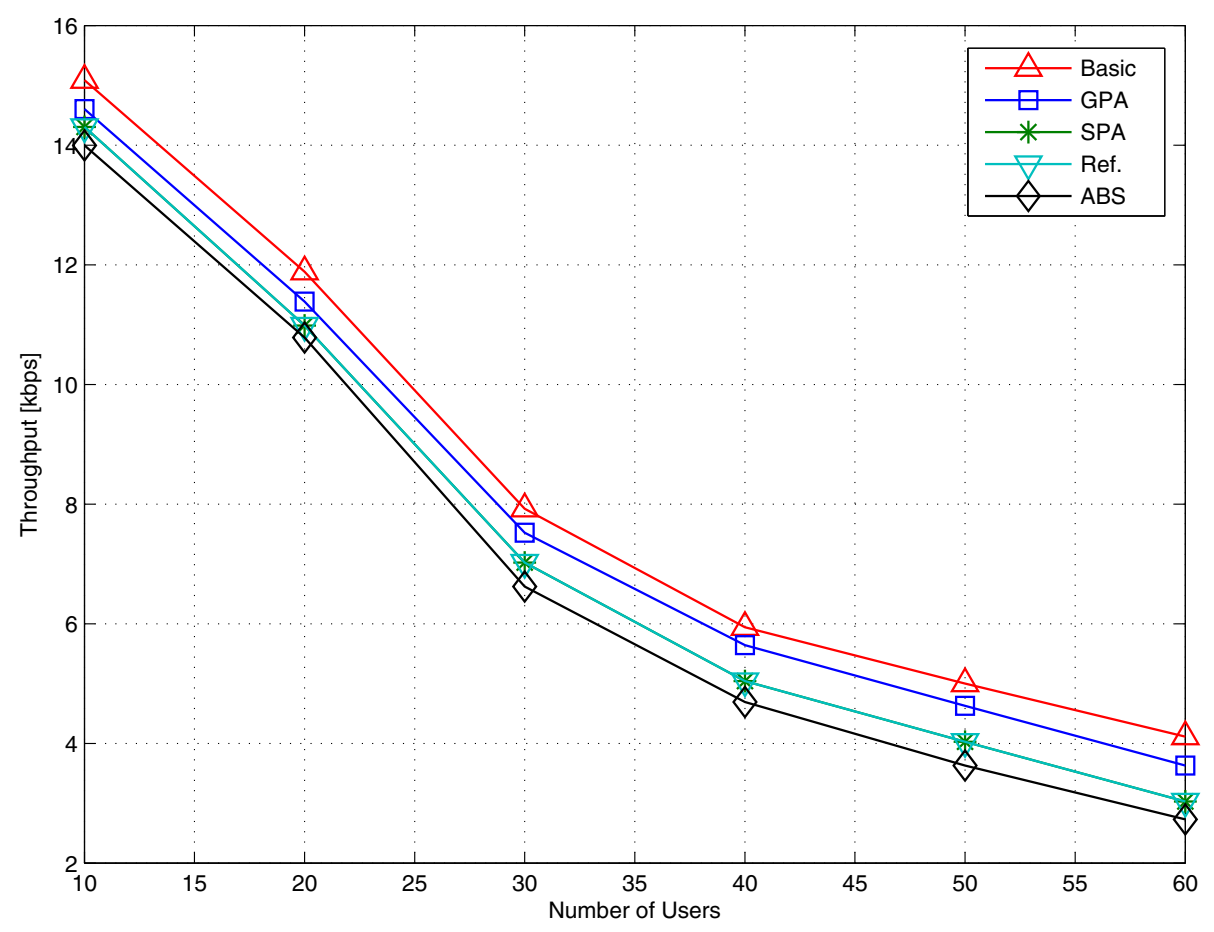

Figure 5 Average per-user throughput of FUEs for CBR flows as a function of the number of MUEs.

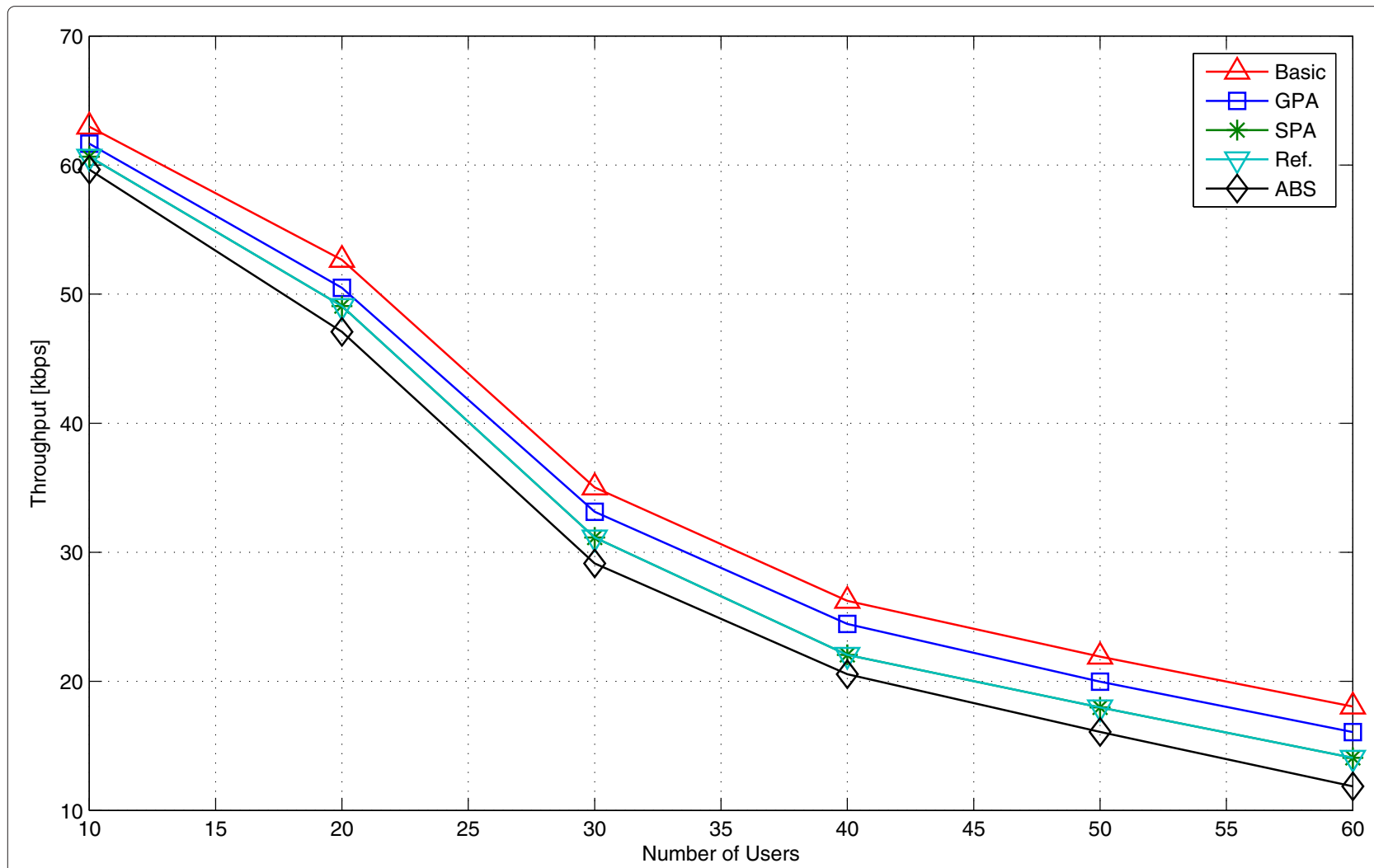

Figure 6 Average per-user throughput of FUEs for VolP flows as a function of the number of MUEs. 


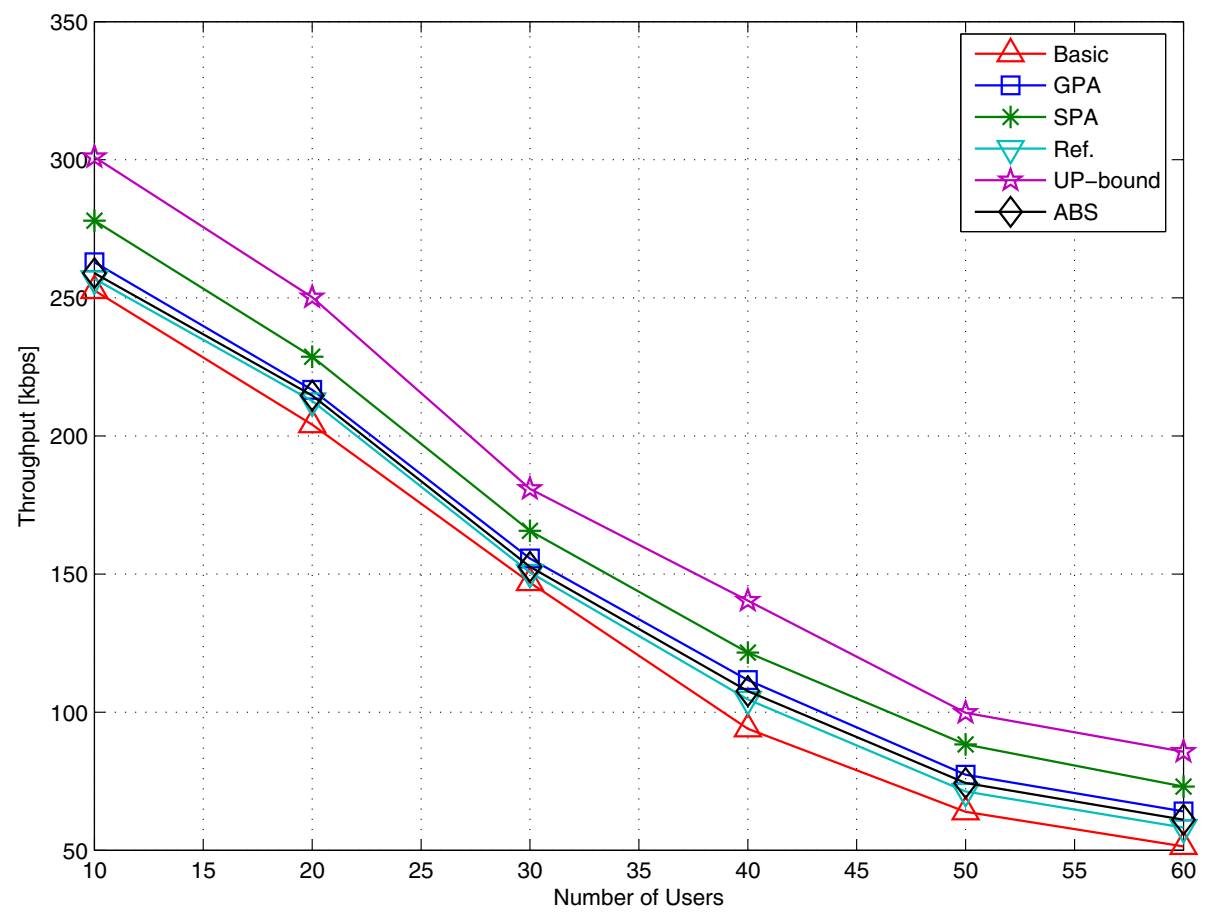

Figure 7 Average per-user throughput of MUEs for video flows.

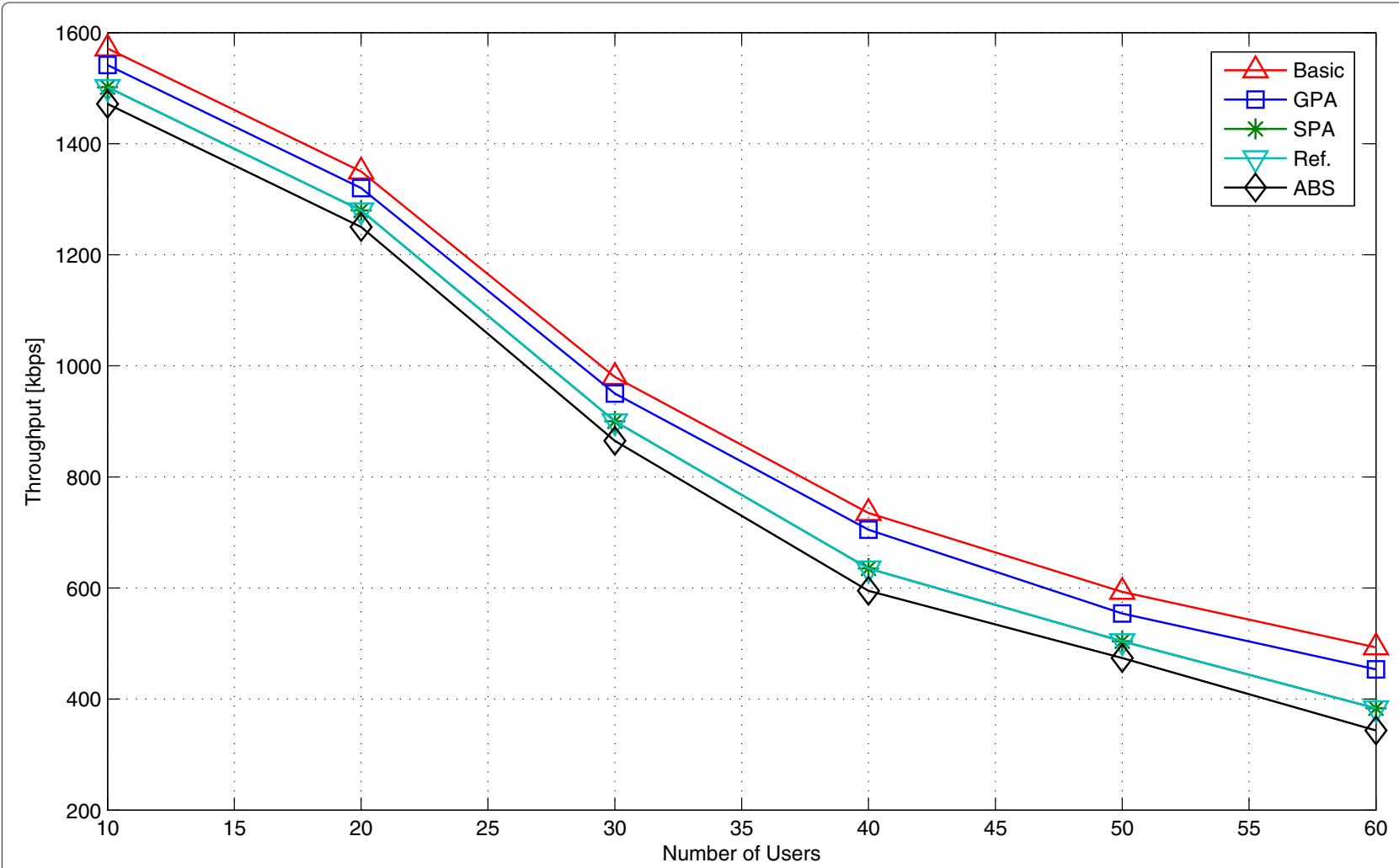

Figure 8 Average per-user throughput of FUEs for video flows as a function of the number of MUEs. 


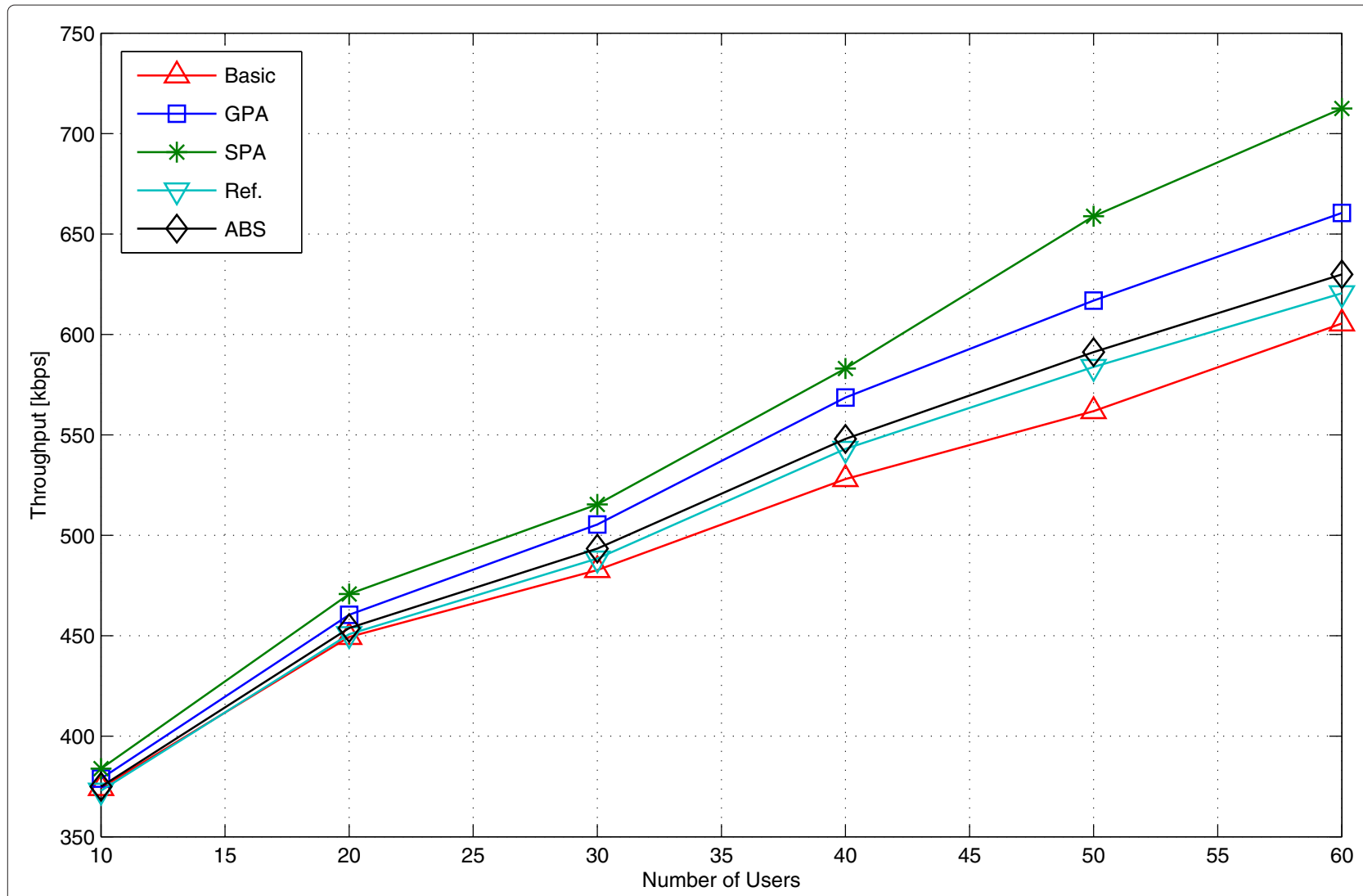

Figure 9 The overall system throughput for Voip flows as a function of the number of MUEs.

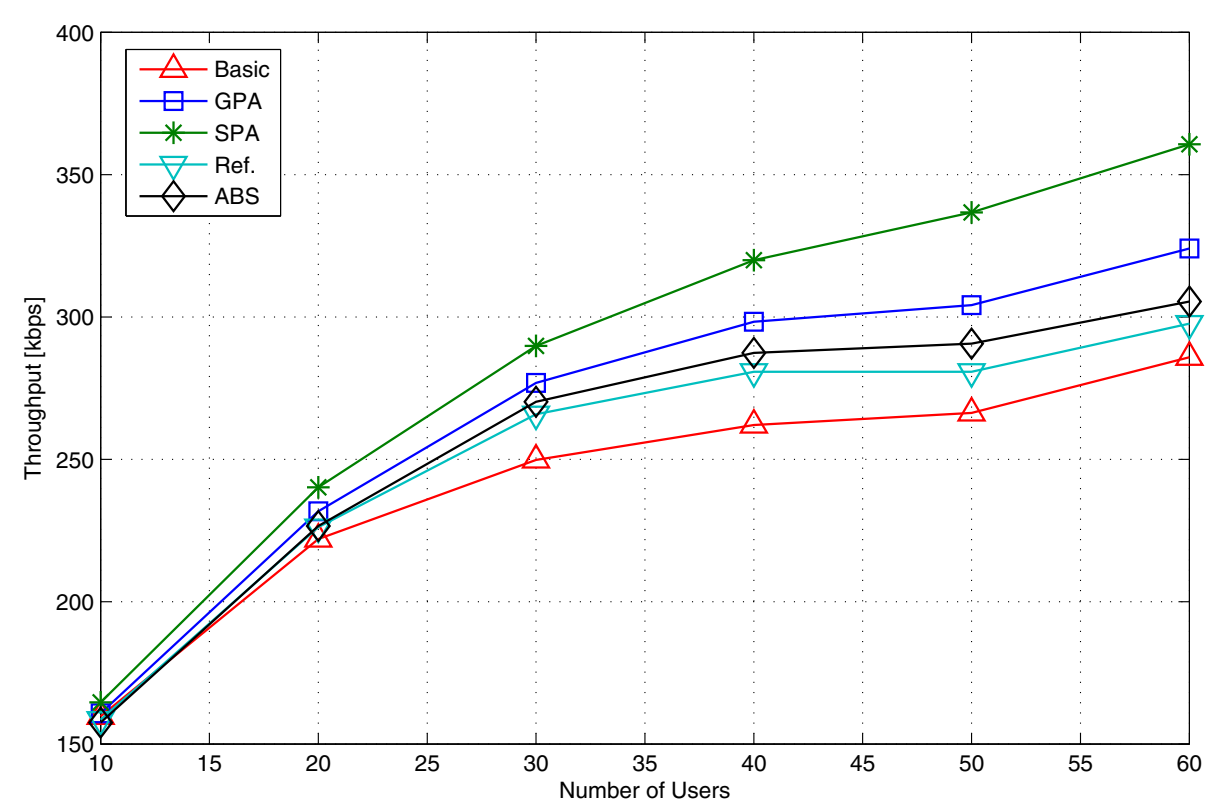

Figure 10 The overall system throughput for CBR flows as a function of the number of MUEs. 
Table 2 Conducted Test Ids and corresponding $\theta_{i}$ values

\begin{tabular}{lcc}
\hline & Test Ids & $\boldsymbol{\theta}_{\boldsymbol{i}}$ values \\
\hline \multirow{2}{*}{ Case A (Priority to FUEs) } & 1 & $(0.1,0.1,0.8)$ \\
& 2 & $(0.2,0.2,0.6)$ \\
\hline & 3 & $(0.25,0.25,0.5)$ \\
Case B (MUEs/FUEs balanced) & 4 & $(0.4,0.1,0.5)$ \\
\hline \multirow{2}{*}{ Case C (Priority to MUEs) } & 5 & $(0.1,0.4,0.5)$ \\
\hline
\end{tabular}

GPA compared to the Basic and Ref. schemes, respectively. Moreover, we observe that the Video traffic is more impacted by the gain in throughput, which is because Video flows usually require additional RBs compared to CBR or VoIP flows given the lower data rate and packet size. The gap between SPA and the MUEs' throughput upper bound is even smaller in the case of Video flows, indicating the validity of the proposed suboptimal solutions. Figure 8 depicts the average per-user throughput with Video flows for FUEs. Here again, our GPA strategy outperforms SPA and Reference schemes.
Finally, Figures 9 and 10 show the overall macrocell and femtocells' system throughput for VoIP and CBR traffics, respectively. In both cases, SPA outperforms GPA, the Reference and ABS schemes, while the Basic scheme offers the lowest performance. SPA strategy achieves the highest performance since it improves the spectral efficiency of MUEs while being agnostic to FUEs' throughput degradation. On the contrary, GPA improves the MUEs' throughput at the expense of a reduced system throughput.

Finally, note that the throughput gain achieved by our proposed techniques is more significant when the density of active UEs increases. Hence, these power adjustment strategies fit perfectly the dense and mobile scenarios of urban areas.

\subsection{Analysis of the impact of the weighting parameters}

In this section, we investigate and analyze the effect of the Score Function weighting parameters $\theta_{i},(i=1,2,3)$ on the performance of our GPA and SPA schemes. As defined in Equation 12, the score of a given interfering FBS $F_{l}$ depends on the values of $\theta_{i}$ parameters which rule the balance between the three context parameters. For a

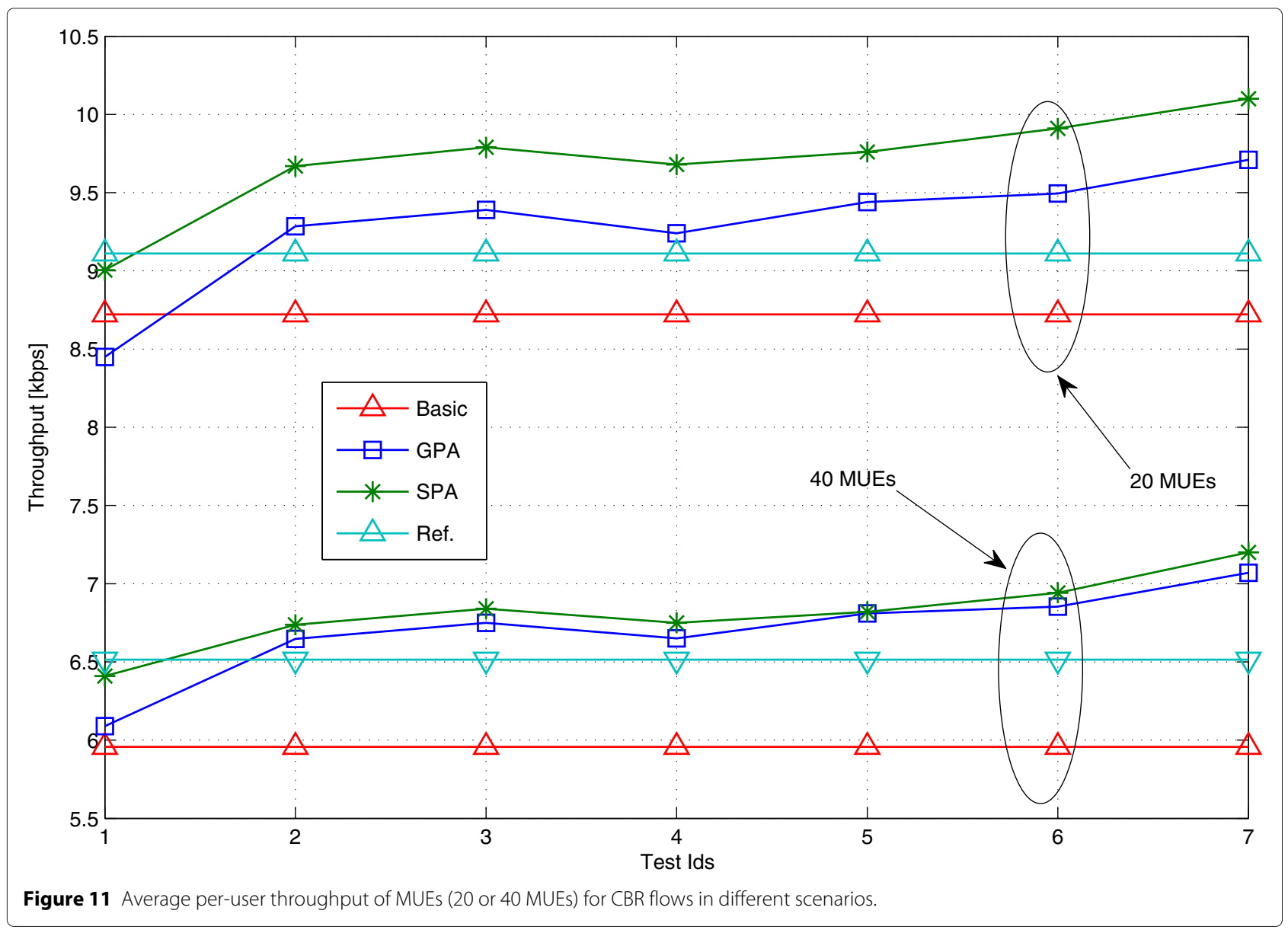


better understanding of the weighting parameters' effect, we recall in the following their respective role in the Score Function:

- $\theta_{1}$ : rules the weight of the 'single interference source' situation as it is associated to the context parameter $R_{F_{l}}$. The single interference situation refers here to the case where the victim MUEs suffer from the interference occurring only from the current FBS $F_{l}$.

- $\theta_{2}$ : acts on the weight of the 'multiple interference sources' situation which indicates that the victim MUEs of sub-set $\mathcal{N}_{F_{l}}$ are suffering from the interference of other FBSs besides $F_{l}$ 's interference.

- $\theta_{3}:$ is used to determine the weight of the outage FUEs on the global $F_{l}$ score. In other words, $\theta_{3}$ manages the degree of awareness of the FUEs' performance within the power adjustment strategy.

It is worth mentioning here that distinguishing the single and multiple interference situations provides a good means to take advanced and adapted power adjustment decisions. For example, the power adjustment strategy may demand more power decrease from FBSs involved in multiple interferences than those creating single interference to rapidly heal the global interference situation. Moreover, note that the association of the $\theta_{i}$ weighting factors and the adopted power adjustment strategy (GPA or SPA) provides two complementary means to realize the best trade-off between MUEs' and FUEs' performance.

To simplify the analysis and discussion, we organize the experiments in three different and significant cases (see Table 2):

1. Case A: corresponds to the scenarios which give a higher priority to FUEs over MUEs while maintaining the same weight between multiple and single interference situations. This case meets the following conditions, $\theta_{1}=\theta_{2}$ and $\theta_{3}>\theta_{1}+\theta_{2}$.

2. Case $B$ : corresponds to the balanced mode where FUEs and MUEs share the same priority. In this case, we have $\theta_{1}+\theta_{2}=\theta_{3}$.

3. Case $C$ : represents the opposite situation of case $A$ since we give more priority to MUEs by setting $\theta_{3}<\theta_{1}+\theta_{2}$.

We evaluate in the following the performance of our power allocation schemes in each of the cases mentioned

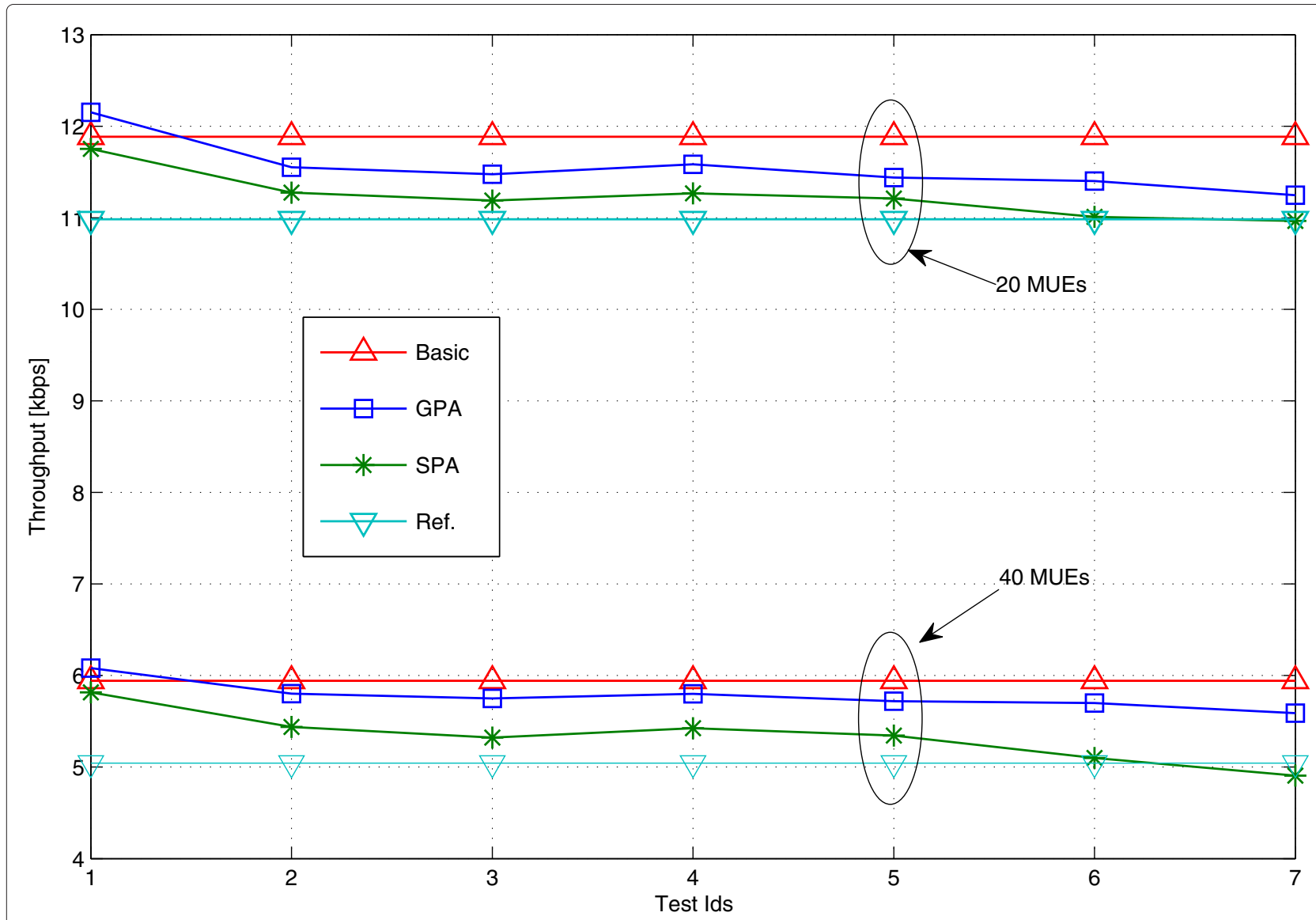

Figure 12 Average per-user throughput of FUEs (20 or 40 MUEs) for CBR flows in different scenarios. 
above. To do so, we assume here CBR traffic flows with 20 and 40 active MUEs using the same simulation parameters provided in Table 1 . The three different cases described above are conducted using the $\theta_{i}$ values provided in Table 2. The average throughput for MUEs and FUEs achieved for each test are presented in Figures 11 and 12, respectively. Note that the Basic and Reference schemes are also depicted in Figures 11 and 12, in order to provide a baseline performance and to have a better insight on how the parameters affect the results compared to the reference schemes. Except for the MUE throughput with Test Id 1, the proposed schemes always outperform the Reference one. Therefore, the following discussion will mainly focus on the weighting parameters' effect on our GPA and SPA strategies.

First of all, comparing the cases where $\theta_{1}+\theta_{2}<\theta_{3}$, i.e., tests 1 and 2, to the cases where $\theta_{1}+\theta_{2}>\theta_{3}$, i.e., tests 6 and 7 , we observe that FUEs are prioritized against MUEs in the first cases and vice versa in the latter ones. Thus, the achieved throughputs are in concordance with the corresponding set of $\theta_{i}$ values, as higher values of $\theta_{1}+\theta_{2}$ enable higher MUE throughput but lower FUE throughput given the constraint $\theta_{1}+\theta_{2}+\theta_{3}=1$. We observe that the value of $\theta_{1}+\theta_{2}$ has more significant effects on the MUE throughput than the value of $\theta_{3}$ on the FUE throughput, given the higher vulnerability of MUEs under FBS interference in our target scenario. Although smaller $\theta_{3}$ implies a lower FUE throughput, Figure 12 shows that there is only a slight decrease, even with the SPA strategy where FBSs are not allowed to increase their power. We can also confirm that a very good trade-off between MUE and FUE throughput is achieved in the balanced case (case B, Test Ids 3,4,5) where $\theta_{1}+\theta_{2}=\theta_{3}$.

Moreover, we study the effect of the discrimination of $\theta_{1}$ and $\theta_{2}$ values, corresponding to different prioritization weights given to MUEs affected by only one femtocell and those affected by several femtocells. These scenarios are represented by Test Ids 4 and 5 in Table 2, assuming $\theta_{1}+\theta_{2}=\theta_{3}$. Clearly, choosing $\theta_{2}>\theta_{1}$ (Test Id 5) provides a higher throughput to MUEs. Thus, giving a larger weight on the interference caused by multiple femtocells allows to decrease their power simultaneously and hence to improve the average MUE throughput. However, the power decrease on those femtocells does not decrease much the FUE average throughput, as shown in Figure 12, validating the efficiency of the proposed power adjustment strategies. In addition, note that compared to the completely balanced scenario (Test Id 3 ), the achieved MUE throughput is further enhanced by selecting $\theta_{2}>\theta_{1}$ (Test Id 5), especially for the GPA strategy for both numbers of MUEs, while keeping the FUE throughput at the same level. This shows the utility of defining distinct $\theta_{1}$ and $\theta_{2}$ values depending on the number of interference sources.
Finally, the results obtained for different sets of $\theta_{i}$ values show that the selected power adjustment strategy, namely, GPA or SPA, along with the appropriate tuning of the $\theta_{i}$ values enable to achieve the required MUE/FUE prioritization level in terms of throughput in an efficient manner.

\section{Conclusions}

This paper has addressed the interference issue in a collocated macro/femtocell HetNets within an urban mobility environment. We proposed femtocell power adjustment methods whose key objective was to increase the average throughput of non-CSG MUEs by limiting the amount of interference caused by femtocells. The main contribution of our approach was to introduce power adjustment parameters whose values are dynamically adapted to the weight of each interfering femtocell through their Score Functions on the global interference situation. Given these weights, the two proposed power adjustment strategies have the common objective of mitigating the interference on victim MUEs while implementing two different degrees of awareness of FUEs' throughput degradation. The simulation results have shown the effectiveness of both proposed strategies in terms of macrocell and femtocell average throughput for different types of traffic. Moreover, it was shown that the proposed priority weights used in the femtocells' Score Functions provide an efficient means for achieving the desired level of macrocell/femtocell throughput trade-off.

\section{Competing interests}

The authors declare that they have no competing interests.

\section{Acknowledgements}

This work was jointly supported by the Grants-in-Aid for bilateral research between France and Japan, co-funded by JSPS and MAEE (PHC Sakura program), the European FP7 Newcom\# NoE, and by the Grants-in-Aid for Scientific Research (KAKENHI) no. 23760334 and no. 26820143 from the Ministry of Education, Science, Sports, and Culture of Japan.

Received: 14 August 2014 Accepted: 10 March 2015

Published online: 06 May 2015

\section{References}

1. E Dahlman, S Parkvall, J Sköld, 3G Evolution: HSPA and LTE for Mobile Broadband, second edition. (Elsevier Academic Press, London, UK, 2008)

2. V Chandrasekhar, JG Andrews, A Gatherer, Femtocell networks: a survey. IEEE Commun. Mag. 46(9), 9-67 (2008)

3. D Lopez-Pérez, A Valcarce, J Zhang, OFDMA femtocells: a roadmap on interference avoidance. IEEE Commun. Mag. 47, 41-48 (2009)

4. Z Wang, W Xiong, C Dong, J Wang, S Li, in International Conference on Computational Problem-Solving (ICCP). A novel downlink power control scheme in LTE heterogeneous network (Leshan, China, 2011), pp. 241-245

5. R Kurda, L Boukhatem, TA Yahiya, in IEEE on Wireless Communications and Networking Systems (WCNC). Interference mitigation in mobile environment through power adjustment in macro and femto cell systems (Istanbul, Turkey, 2014)

6. R Kurda, L Boukhatem, TA Yahia, M Kaneko, in IEEE Symposium on Computers and Communications (ISCC). Power adjustment mechanism using context information for interference mitigation in two-tier heterogeneous networks (Madeira, Portugal, 2014) 
7. MS Jin, S Chae, DI Kim, in Proc. IEEE VTC 11-Spring. Per cluster based opportunistic power control for heterogeneous networks (Budapest, Hungary, 2011)

8. P Gao, D Chen, M Feng, D Qu, T Jiang, in IEEE on Wireless Communications and Networking Conference (WCNC). On the interference avoidance method in two-tier LTE networks with femtocells (Shanghai, China, 2013), pp. 3585-3590

9. D Lopez-Pérez, A Valcarce, A Ladanyi, J Zhang, Intracell handover for interference and handover mitigation in OFDMA two-tier macrocell-femtocell networks. EURASIP J Wireless Commun. Netw. 10 (2009)

10. Y Bai, J Zhou, L Liu, L Chen, H Otsuka, in IEEE International Symposium on Personal, Indoor and Mobile Radio Communications (PIMRC). Resource coordination and interference mitigation between macrocell and femtocell (Tokyo, Japan, 2009), pp. 1401-1405

11. F Bernardo, R Agustí, J Cordero, C Crespo, in Sixth Advanced International Conference on Date of Conference: Telecommunications (AICT). Self-optimization of spectrum assignment and transmission power in OFDMA femtocells (Barcelona, Spain, 2010), pp. 404-409

12. M Kim, H Won, FA Je Tobagi, in IEEE Global Telecommunications Conference (GLOBECOM). Cross-tier interference mitigation for two-tier OFDMA femtocell networks with limited macrocell information (Miami, Florida, USA, 2010), pp. 1-5

13. M Bennis, L Giupponi, EM Diaz, M Lalam, M Maqbool, EC Strinati, A De Domenico, M Latva-aho, in IEEE Wireless Communication, Vehicular Technology, Information Theory and Aerospace \& Electronic Systems Technology (Wireless VITAE). Interference management in self-organized femtocell networks: the BeFEMTO approach (Chennai, India, 2011), pp. 1-6

14. T Nakano, M Kaneko, K Hayashi, H Sakai, in Proc. of IEEE VTC-Spring. Interference mitigation based on partial CSI feedback and overhearing in an OFDMA heterogeneous system (Dresden, Germany, 2013), pp. 1-5

15. T Nakano, M Kaneko, K Hayashi, H Sakai, in IEEE International Symposium on Personal, Indoor and Mobile Radio Communications (PIMRC). Downlink power allocation with CSI overhearing in an OFDMA macrocell/femtocell coexisting system (Sydney, Australia, 2012), pp. 454-459

16. A Zalonis, N Dimitriou, A Polydoros, J Nasreddine, P Mähönen, in IEEE Wireless Communications and Networking Conference (WCNC). Femtocell downlink power control based on radio environment maps (Paris, France, 2012), pp. 390-394

17. 3GPP, Simulation assumptions and parameters for FDD HeNB RF requirements, 3GPP TSG RAN WG4 R4-092042 (May 2008). http://www. 3gpp.org/ftp/Specs

18. Beek van de J, T Cai, S Grimoud, B Sayrac, P Mähönen, J Nasreddine, J Riihijärvi, How a layered REM architecture brings cognition to today's mobile networks. IEEE Wireless Commun. 19(4), 17-24 (2012)

19. G Piro, LA Grieco, G Boggia, F Capozzi, P Camarda, Simulating LTE cellular systems: an open source framework. IEEE Trans. Vehic. Technol. 60, 498-513 (2010)

20. X Li, L Qian, D Kataria, in Proc. of the Conference on Information Sciences and Systems (CISS). Downlink power control in co-channel macrocell femtocell overlay (Baltimore, USA, 2009)

21. G Cao, D Yang, X Ye, X Zhang, in IEEE on Wireless Communications and Networking Conference (WCNC). A downlink joint power control and resource allocation scheme for co-channel macrocell-femtocell networks (Beijing, China, 2011), pp. 281-286

22. R Madan, J Borran, A Sampath, N Bhushan, A Khandekar, T Ji, Cell association and interference coordination in heterogeneous LTE-A cellular networks. IEEE J. Selected Areas Commun. 28(9), 1479-1489 (2010)

23. B Sayrac, D2.4: Final system architecture, deliverable of FARAMIR project, (December 2011). available at http://www.ict-faramir.eu/

24. L Wang, Y Zhang, Z Wei, in Proceedings of the 69th IEEE Vehicular Technology Conference (VTC 09). Mobility management schemes at radio network layer for LTE femtocells (Barcelona Spain, 2009)

25. C Chen-Nee, RH Katz, in IEEE International Communication (ICC). Characterizing packet audio streams from Internet multimedia applications, vol. 2 (New York City, USA, 2002), pp. 1199-1203

26. Video trace library. http://trace.eas.asu.edu/

27. CATT, Evaluations of RSRP/RSRQ measurement (R4-110284), (Austin, TX, 2011)

\section{Submit your manuscript to a SpringerOpen ${ }^{\circ}$ journal and benefit from:}

- Convenient online submission

- Rigorous peer review

- Immediate publication on acceptance

- Open access: articles freely available online

- High visibility within the field

- Retaining the copyright to your article

Submit your next manuscript at $>$ springeropen.com 